\title{
Modeling and Forecasting Morbidity and Disease-mortality of Chinese Impaired Lives with the General Chronic Diseases
}

\author{
Ning ZHANG \\ Central University of Finance and Economics, Beijing 100081 \\ Email: nzhang@amss.ac.cn \\ Yanran YUAN \\ Central University of Finance and Economics, Beijing 100081 \\ Email: yuanxiao1124@gmail.com
}

\begin{abstract}
The paper did some work to analyze the longevity risk of impaired lives with general chronic diseases. The work was based on the age-specific, time-specific and disease-specific data set which is from the underwriting data base, the Chinese healthy yearbook and the medical institution. After some analysis on the final data, the results showed that there was an obvious upward trend in morbidity and downward trend mortality. All the results can be used in the pricing process of insurance products for impaired lives. The paper also showed the pricing results of innovative life insurance products for impaired lives with diabetes. The conclusion means a good market chance for any insurance companies.
\end{abstract}

Keywords impaired lives; general chronic diseases; Lee-Carter model

\section{Introduction}

Although longevity risk has attracted our attention, normal group which means the healthy or standard group is the only object of most studies. And a direct question will be asked that how the longevity risk of Chinese impaired lives is. We can't neglect this question since there are a large amount of impaired lives. From this view, it is better to understand Chinese longevity risk.

Of course, it is consistent with the insurance market. Our insurance products like life insurance products are for the normal lives. For the impaired lives, the market hopes they have bought healthy insurance products when they are normal lives. But it is ridiculous for impaired lives to forecast their worse physical state ${ }^{[1]}$. So should we exclude them to buy the life insurance products? To answer this question, we must know how much the risk is in the view of an insurance company is. We should find the mortality of the impaired group and the morbidity of diseases.

The followings are some perspectives why the risk of impaired lives including longevity risk become our concerns.

Received November 21, 2013, accepted January 3, 2014

Supported by MOE Project of Key Research Institute of Humanities and Social Sciences in Universities (Grant No. 11JJD790004), Youth Innovation Group of CUFE and Disaster Research Fund of PICC 


\subsection{Chinese general chronic diseases}

Recently, more and more Chinese people pay attention to their health problems. Some reports have showed that the increased work pressure and busy life style have affect health. Maybe there are some arguments, but the hope away from diseases is universal for any people.

Taking general chronic diseases as an example, the statistical results remind us of more instructive information. That is the growing morbidity rate of the general chronic disease. Just from the newspaper, the number of potential diabetes is about 150 million and the number in treating course is 92 million now (male 50 million and female 42 million), morbidity rate is $9.7 \%$, but the number was 78 million about ten years ago. The total population growing rate can't explain it.

In medical field, the general chronic diseases include the mental disease, the cardiovascnlar and cerebrovascular diseases, chronic cancer, chronic respiratory disease and diabetes. Some statistical materials also gave that a large amount of Chinese people is tortured by the general chronic diseases. The following gives some surveys both in the disease view and in the time view.

Table 1 Some surveys of several chronic diseases

\begin{tabular}{lcccc}
\hline Disease & Total & Severe & Statistical year Statistical sources \\
\hline Cardiovascnlar and & 170 million $^{1}$ & 17 million & 2004 & Chinese ministry of health \\
cerebrovascular diseases & & & \\
Mental disease & 100 million & 16 million & 2006 & Chinese centers of disease control \\
Chronic respiratory $^{20 \text { million }}$ & 20 million & 2008 & \\
disease $^{2}$ & & & & \\
Choric cancer $^{3}$ & About 10 million & 8 million & 2010 & Cancer registration center of China \\
Diabetes $^{4}$ & 148 million & 92 million & 2012 & New England Journal of Medicine \\
\hline
\end{tabular}

Remark 1 Here 170 million is the number of people with high blood pressure since it is the direct cause to cardiovascnlar and cerebrovascular diseases.

Remark 2 Here only is the statistical result of chronic bronchitis.

Remark 3 The total number is estimated by related research since it is difficult to find potential cancer patients. But the report also gave some other important result. Chinese cancer registration year book shows that the morbidity of cancer growing by $3 \% \sim 5 \%$ one year and 3.15 million people are diagnosed cancer one year.

Remark 4 The result was from a paper in New England Journal of Medicine, written by several specialists who worked in several famous hospitals in China.

\subsection{Insurance products market}

This is a huge market for the life insurance which has been ignored in the last 20 years. Furthermore, considering all the impaired lives with different chronic diseases, the number is about 300 million which is almost the population size of US. So it is a huge insurance market for insurance company and it is an important group for the government ${ }^{[2]}$.

In Chinese insurance market, both the life insurance products and health insurance products 
are for the normal people without uncovered diseases although a few of impaired lives can be covered by increasing premium ${ }^{[3]}$. The increasing premium is not the actuarial results but the result of normal premium multiplying by a coefficient. It is not responsible for both insurers and policy holders. Even that, there are many impaired lives who can't be accepted by the insurance companies. So how do they transfer the mortality risk or can they get some compensation when they died? It is reasonable just as the normal people do. And we also observed that the similar insurance demand is much stronger than the normal lives. The reason is direct that they face the greater $\operatorname{risk}^{[4-5]}$.

For getting a persuasive result, we make a simple survey through a cooperated medical hospital. The samples are all patients with diabetes and the number is 532. A survey questionnaire with 10 questions was made. All the questions are about demand and advices of insurance products for impaired lives.

We choose a typical life insurance product which can be found in many insurance companies. The coverage is 400 thousands. When an investigation began, we introduce the simple life insurance knowledge without any inducing information. After that, we will take their patience to finish the form. Several questions is related with demands, such as the followings: Do you need the introduced life insurance products? If the premium is more than the normal premium, 1.3 times of normal premium, can you accept it? If the premium is 1.5 times of normal premium, can you accept it? Could you tell us what is the most reason to push you make this decision?

At last, we obtained 508 effective results after simple statistical calculation and it included 370 male samples and 238 samples. Table 2 gave a simple survey result of this investigation. It is obvious that the insurance demand of impaired lives is more than that of normal lives.

Table 2 Simple survey results of insurance demand (diabetes)

\begin{tabular}{lccc}
\hline & Male & Female & Total \\
\hline Life insurance demand & 292 & 156 & 448 \\
1.3 accepted & 271 & 114 & 385 \\
1.5 accepted & 223 & 79 & 302 \\
\hline
\end{tabular}

Always some people hesitate to the cost of impaired insurance products introduction. In fact, most insurance companies have accumulated much of experience of normal products. The pricing technique, the commission management and sale channel can be used in this new business. So it is a good market chance for the insurance companies.

\subsection{Longevity views}

Longevity risk become a general risk for any country in the world. Of course, we can observe the downwards mortality of Chinese people although the longevity data set has many problems like missing data, outliers and so on. But for a huge amount of population, it is necessary to consider different groups, like people in city or people in the rural area. Does the longevity risk exist in these subdivided group?

And the people with chronic diseases is first to consider since its large number and strong insurance demands. When the insurance markets have accepted the group, the longevity risk will come to the managers. Another fact also remind us of the impact of longevity that the 
intrinsic risk is more than the normal group. For insurance companies, maybe the longevity risk can be applied to hedging the other risk. Just like what we said, the risk is chance. Always, longevity risk means the risk with time. In other words, how much is the impact of time on the mortality. From the total population, the impact on the birth can't be ignored. Both the birth rate and the mortality will bring the influence on the population. For the group with chronic diseases, the birth means morbidity whose result tell us how many normal people have enter the impaired group. So the longevity risk of impaired lives includes the analysis on the morbidity.

The article includes four parts: the first part is the data mining and to calculate the morbidity and normal mortality; the second part is to model and forecast the morbidity; the third part is to calculate the disease-mortality, and to model and forecast disease-mortality; the fourth part used the results to price the life insurance products for the impaired lives.

\section{Data and statistical work}

Our data sources include three parts: Chinese health statistical yearbook, the underwriting database from insurance companies and the medical data. The final data set was obtained step by step. Here the final data set means the two-dimensional data form or matrix for one type of disease. Any value in the matrix means the mortality or morbidity at specific age and in the specific year. So the total final data set is three-dimensional, age-specific, year-specific and disease-specific.

\subsection{Calculating morbidity}

Our work started from the underwriting data base since it is the most actual information of policyholders. But there is no statistical data of mortality statistics of impaired lives, we calculated the mortality of impaired lives according to the collected policy data of critical illness insurance and relevant data from Ministry of Health of China.

We collected policy data from a life insurance company, and selected out policy data of critical illness insurance from 1999 to 2011, 13 years totally. We used actuarial moment estimation to calculate morbidity. Morbidity of a kind of critical illness was equal to the ratio of total number of claims of the critical illness insurance to total actuarial exposures of the critical illness insurance insured. For the critical illness insurance insured, in unit time (one year), if the policies are effective, over or withdrawn, their actuarial exposures are their real exposures, and if the claims are over in the year, the actuarial exposure is one year.

The procedure of calculating morbidity is showed below.

The first step, we needed to build a underwriting-claim database of critical illness insurance from the life insurance company's policy data. First selected out the items that were needed in morbidity calculation from policy underwriting data such as insurance type, underwriting date, age, rider, birthday and policy claim data such as insurance type, claim date, claim type, claim reason, rider, settle type to build a underwriting database and a claim database separately. After that, we selected out critical illness insurance policies both from the underwriting database and claim database got above. Then joined the underwriting database and claim database of critical illness insurance together, and added items which were needed to calculate morbidity, such as exit date, exit reason, policy year, policy date, number of claims, exposures and so on. 
The second step, for one year between 1999 and 2011, divided the year into two periods by the policy date where supposed that the policy date was consistent with the insured's birthday. In each of the two periods, added policy year 1, age 1, number of claims 1, exposure 1 and policy year 2 , age 2 , number of claims 2 , exposure 2 separately. There were also some adjustments needed to be done, such as adjustment of exit date, and if one policy has more than one claims, only one claim needed to be accounted. Then we calculated each policy's number of claims 1 , exposure 1 and number of claims 2, exposure 2 in each period of the year, when calculated exposures, the actuarial exposures should be used. After that, we summed up number of claims and exposures which were grouped by age separately, so we can get number of claims and exposures of different ages in the year, and the ratio of the two results was just the morbidity we need.

For each year from 1999 to 2011, we can repeat the processing method above and we can get numbers of claims, exposures and morbidity of different ages in every year. We can also sum up numbers of claims and exposures grouped by age of the 13 years and get morbidity of each age for the 13 years together. The third step, we can also repeat above two steps for different critical illness. When morbidity of different critical illness is calculated, it needed to separate the disease into several big types because of not enough data, for example, when it mentioned cancer, it meant a collection of thyroid cancer, breast cancer, cervical cancer and other species. After critical illness separation, the article calculated morbidity results of cancer and diabetes as the way showed above.

After getting the morbidity of different critical illness, we found that for some diseases, the morbidity results were not accurate enough because of little amount of policy data. According to the life insurance company's experience, when the number of claims is less than 250 , the company thinks that the morbidity is not accurate enough. So when we got the morbidity results of cancer and diabetes, both of the two diseases had some age bands which had inaccurate results, such as 0 to 24 and 60 to 74 years old for cancer, so only morbidity results of 25 to 59 years old can be used to calculate cancer patients' mortality in the below.

\subsection{Calculating mortality}

As there is no statistical data of morality of impaired lives, we calculated mortality of impaired lives according to the morbidity which was calculated according to a life insurance company's critical illness insurance policy data and mortality statistical data from Ministry of Health of China. But there was a question that the statistical calibers of the two kinds of data were different, one was calculated on the basis of the insured of a life insurance company's critical illness insurance, and the other was counted on the basis of the whole urban residents of our country.

To solve this problem, we had done some adjustments to the morbidity results with Taiwan residents' morbidity of cancer and diabetes. The method to adjust the insured's morbidity was that the new morbidity which would be used to calculate mortality of impaired lives was equal to the sum of the insured's morbidity multiplied by weight one and Taiwan residents' morbidity multiplied by weight two, where the weight one was equal to the ratio of the number of critical illness insurance insured to the sum of the number of insured and the population of Taiwan, and the weight two was equal to 1 minus weight one. When calculating weight one, we used the 
mean of the insured's exposures from 1999 to 2011 as the number of critical illness insurance insured, and the average of Taiwan population from 2005 to 2007 as the population of Taiwan.

For some chronic diseases we focus on, the adjustment is not enough to get the final 3dimentional data set. We continued to use medical research and survey to fit and modify the above results. The Patient Tracing Division accumulated a large amount of information from patients with diabetes and chronic cancer (some selected types which seems to be modest). The data from medical institution is from 1995 to 2010. And most of data is for the age above 35 , only some cancer are for the age above 23. Since the policyholders of insurance companies whose data we use are come from cities, we use city part of the data from medical institution and the city surveys in the Chinese healthy yearbook. In other word, our research is based on Chinese urban data and get the conclusion of Chinese urban impaired lives. Of course, the rural research is on the research way for us. Here, we also use some results from the public medical reports, like Chinese diabetes survey.

\section{Some results of the morbidity and mortality}

The following table (table 3 ) showed the morbidity of diabetes and chronic cancers (limited cancer). And table 4 showed the mortality of diabetes and chronic cancers. Figure 1 gives a comparing result between the normal lives and the impaired lives with diabetes.

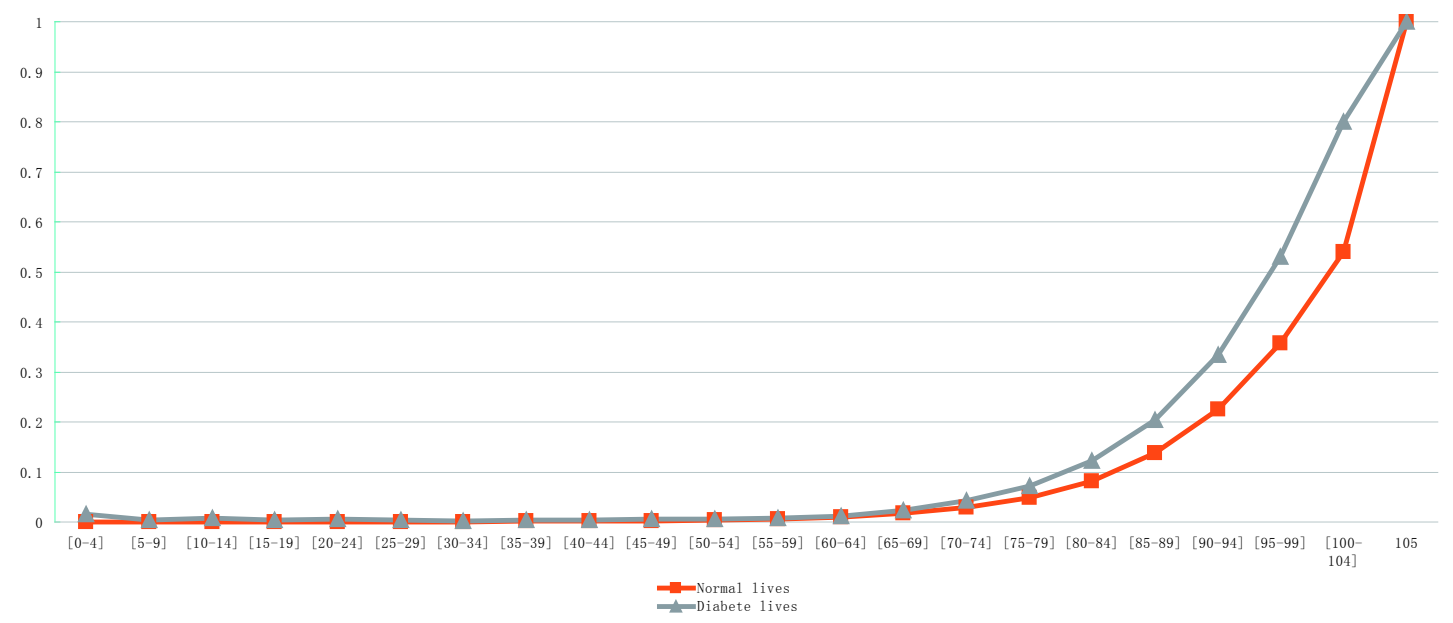

Figure 1 comparison between normal lives and impaired lives with diabetes

The Lee-Carter model $m_{x, t}=A_{x}+B_{x} \times K_{t}$ can be used for analysis on the morbidity of diabetes. Table 5 gave the analyzing result of $K_{t}{ }^{[6]}$. And the Table 6 gave the forecasting result of $K_{t}$. They both show us an fixed trend of mortality of diabetes. It is consistent with the Diabetes Survey Reports (2011). But our results showed a less trend than that in some existing reports $^{[7-8]}$. 
Table 3 Morbidity of chronic cancers and diabetes

\begin{tabular}{ccc}
\hline & Limited Cancer & Diabetes \\
\hline$[30-34]$ & $0.02 \%$ & $0.00 \%$ \\
{$[35-39]$} & $0.03 \%$ & $0.00 \%$ \\
{$[40-44]$} & $0.06 \%$ & $0.00 \%$ \\
{$[45-49]$} & $0.10 \%$ & $0.00 \%$ \\
{$[50-54]$} & $0.17 \%$ & $0.01 \%$ \\
{$[55-59]$} & $0.25 \%$ & $0.01 \%$ \\
{$[60-64]$} & $0.36 \%$ & $0.03 \%$ \\
{$[65-69]$} & $0.55 \%$ & $0.06 \%$ \\
{$[70-74]$} & $0.85 \%$ & $0.11 \%$ \\
{$[75-79]$} & $1.18 \%$ & $0.18 \%$ \\
{$[80-84]$} & $1.45 \%$ & $0.24 \%$ \\
{$[85+]$} & $1.56 \%$ & $0.30 \%$ \\
\hline
\end{tabular}

Table 4 Mortality of chronic cancers and diabetes

\begin{tabular}{lcc}
\hline & Limited cancer & Diabetes \\
\hline$[25-29]$ & $0.02 \%$ & $0.04 \%$ \\
{$[30-34]$} & $0.04 \%$ & $0.07 \%$ \\
{$[35-39]$} & $0.06 \%$ & $0.11 \%$ \\
{$[40-44]$} & $0.11 \%$ & $0.18 \%$ \\
{$[45-49]$} & $0.17 \%$ & $0.28 \%$ \\
{$[50-54]$} & $0.24 \%$ & $0.41 \%$ \\
{$[55-59]$} & $0.34 \%$ & $0.53 \%$ \\
{$[60-64]$} & $0.44 \%$ & $0.68 \%$ \\
{$[65-69]$} & $0.54 \%$ & $0.82 \%$ \\
{$[70-74]$} & $0.08 \%$ & $1.20 \%$ \\
\hline
\end{tabular}

Table 5 The analyzing result of $K_{t}$

\begin{tabular}{cc}
1995 & $-\mathbf{0 . 8 2}$ \\
1996 & -0.659 \\
1997 & -0.5 \\
1998 & -0.329 \\
1999 & -0.167 \\
2000 & -0.008 \\
2001 & 0.156 \\
2002 & 0.315 \\
2003 & 0.484 \\
2004 & 0.646 \\
2005 & 0.805 \\
2006 & 0.97 \\
2007 & 1.149 \\
2008 & 1.325 \\
2009 & 1.512 \\
2010 & 1.692 \\
\hline
\end{tabular}

Table 6 The forecasting result of $K_{t}$

\begin{tabular}{lc}
2009 & $\mathbf{1 . 5 1 2}$ \\
2010 & 1.692 \\
2011 & 1.86 \\
2012 & 2.031 \\
2013 & 2.2 \\
2014 & 2.372 \\
2015 & 2.553 \\
2016 & 2.726 \\
2017 & 2.896 \\
2018 & 3.07 \\
2019 & 3.242 \\
2020 & 3.413 \\
\hline
\end{tabular}

The diabetes is not a special case. In the five chronic diseases, we find the similar trend except chronic respiratory disease which showed an almost-horizontal trend. We may make a conclusion that the morbidity of general chronic diseases shows a clearly stable linearly increasing trend over time, the rising trend is more obvious as ages increase. The same work was to do with the mortality for Diabetes. Table 7 showed the analyzing results and forecasting results of $K_{t}$. 
Table $7 \quad K_{t}$ of mortality for diabetes

\begin{tabular}{ll}
1995 & 5.018299 \\
1996 & 3.879055 \\
1997 & 2.760921 \\
1998 & 1.613643 \\
1999 & 0.515753 \\
2000 & -0.60656 \\
2001 & -1.67137 \\
2002 & -2.85782 \\
2003 & -3.96167 \\
2004 & -5.06286 \\
2005 & -6.194 \\
2006 & -7.31253 \\
2007 & -8.43757 \\
2008 & -9.57973 \\
2009 & -10.7092 \\
2010 & -11.8456 \\
2011 & -12.88 \\
2012 & -14.02 \\
2013 & -15.17 \\
2014 & -16.29 \\
2015 & -17.41 \\
2016 & -18.54 \\
2017 & -19.67 \\
2018 & -20.76 \\
2019 & -21.88 \\
2020 & -23.00 \\
\hline &
\end{tabular}

Table 8 The net premium

\begin{tabular}{cccc}
\hline Period & Age 30 & Age 40 & Age 50 \\
\hline 1 & 0.003 & 0.004 & 0.006 \\
2 & 0.005 & 0.009 & 0.012 \\
3 & 0.008 & 0.012 & 0.017 \\
4 & 0.01 & 0.016 & 0.022 \\
5 & 0.012 & 0.02 & 0.027 \\
6 & 0.015 & 0.024 & 0.033 \\
7 & 0.017 & 0.027 & 0.039 \\
8 & 0.019 & 0.031 & 0.044 \\
9 & 0.022 & 0.034 & 0.05 \\
10 & 0.024 & 0.037 & 0.054 \\
11 & 0.026 & 0.041 & 0.061 \\
12 & 0.029 & 0.044 & 0.068 \\
13 & 0.031 & 0.047 & 0.074 \\
14 & 0.033 & 0.05 & 0.079 \\
15 & 0.035 & 0.053 & 0.085 \\
16 & 0.038 & 0.056 & 0.094 \\
17 & 0.04 & 0.06 & 0.103 \\
18 & 0.042 & 0.063 & 0.111 \\
19 & 0.044 & 0.066 & 0.118 \\
20 & 0.046 & 0.069 & 0.125 \\
\hline
\end{tabular}

We can also find the longevity trend in the mortality of impaired lives.

\section{Actuarial present value of impaired life insurance products}

Here we gave a simple net premium results of life insurance for impaired lives with diabetes at different ages based on the following formula ${ }^{[9]}$. It is computed based on 2008's data and can be adjusted by the trend of mortality. Table 8 showed this. Considered the insurance demands in the first part of the paper, it is a gold market chance.

$$
P=Q \sum_{m=0}^{n-1} m \mid q_{x}^{*} v^{m+1}=Q v^{*} q_{x}+Q v^{2 *} p_{x} q_{x+1}+\cdots+Q v_{n-1}^{n *} p_{x} q_{x+n-1}+\cdots
$$




\section{Conclusion}

We use the classic Lee-Carter and other derivative models to analyze the morbidity and mortality of impaired lives with general chronic diseases. In the results, we got some results. 1) Classical Lee-Carter model is enough for this. 2) The morbidity has obvious upward trend for the population. Although many reasons can explain it, we should pay more attention to our health. 3) From the result, we find that longevity risk is obvious for the impaired lives with the four chronic diseases. It changed the viewpoints which were hold by many people in medical field. We compared the longevity risk for the impaired lives with the longevity risk for the healthy lives and found some results like this: for the diabetes, the former is sharper, about 2.27 to 1.98 , but for the other two chronic diseases, the trend is similar with the normal longevity risk.

All the results were used to price the life insurance products for the impaired lives. The pricing result is a useful and important advice for the insurance company. Just as we know, only several similar products are sold in Chinese mainland and most of them were priced based on the method of multiplying a coefficient directly.

Acknowledgements We thank the referees for their time and comments.

\section{References}

[1] Brian Ridsdale: Underwriting enhanced and substandard annuities in the UK. LA: A World of Mortality Issues and Insights Seminar, 2012.

[2] Wilmoth J R. Computational methods for fitting and extrapolating the Lee-Carter model of mortality change. Berkeley: Technical report, 1993.

[3] Raws A. Overview of reserving practices for substandard life policies. North American Actuarial Journal, 1998(4): 102-108.

[4] Ainslie R. Annuity and insurance products for impaired lives. UK: Staple Inn Actuarial Society, 2000: 41-49.

[5] Cox S H, Lin Y J. Natural hedging of life and annuity mortality risks. North American Actuarial Journal, 2007, 11(3): 1-15.

[6] Lee R D, Carter L R. Modeling and forecasting US mortality. Journal of the American Statistical Association, 1992, 87(419): 659-671.

[7] Renshaw A E, Haberman S. On simulation-based approaches to risk measurement in mortality with specific reference to Poisson Lee-Carter modeling. Insurance: Mathematics and Economics, 2008, 42: 797-816.

[8] Koissi M, Shapiro A F, Hognas G. Evaluating and extending the Lee-Carter model for mortality forecasting: Bootstrap confidence internal. Insurance: Mathematics and Economics, 2006, 38(1): 1-20.

[9] Zhang N. Introduction and computation of longevity risk index based on mortality rate decomposition model. Springer Communications in Computer and Information, 2012, 289: 608-615. 